University of Nebraska - Lincoln

DigitalCommons@University of Nebraska - Lincoln

\title{
Gypenosides Reduced the Risk of Overweight and Insulin Resistance in C57BL/6J Mice through Modulating Adipose Thermogenesis and Gut Microbiota
}

Jie Liu

Shanghai Jiao Tong University

Yanfang Li

Shanghai Jiao Tong University

Puyu Yang

Shanghai Jiao Tong University

Jianchun Wan

Shanghai Jiao Tong University

Qimeng Chang

Fudan University Minhang Hospital

See next page for additional authors

Follow this and additional works at: https://digitalcommons.unl.edu/usdaarsfacpub

Liu, Jie; Li, Yanfang; Yang, Puyu; Wan, Jianchun; Chang, Qimeng; Wang, Thomas T. Y.; Lu, Weiying; Zhang, Yaqiong; Wang, Qin; and Yu, Liangli Lucy, "Gypenosides Reduced the Risk of Overweight and Insulin Resistance in C57BL/6J Mice through Modulating Adipose Thermogenesis and Gut Microbiota" (2017). Publications from USDA-ARS / UNL Faculty. 1800.

https://digitalcommons.unl.edu/usdaarsfacpub/1800

This Article is brought to you for free and open access by the U.S. Department of Agriculture: Agricultural Research Service, Lincoln, Nebraska at DigitalCommons@University of Nebraska - Lincoln. It has been accepted for inclusion in Publications from USDA-ARS / UNL Faculty by an authorized administrator of DigitalCommons@University of Nebraska - Lincoln. 


\section{Authors}

Jie Liu, Yanfang Li, Puyu Yang, Jianchun Wan, Qimeng Chang, Thomas T. Y. Wang, Weiying Lu, Yaqiong Zhang, Qin Wang, and Liangli Lucy Yu 


\title{
Gypenosides Reduced the Risk of Overweight and Insulin Resistance in C57BL/6J Mice through Modulating Adipose Thermogenesis and Gut Microbiota
}

\author{
Jie Liu, ${ }^{\dagger \neq}$ Yanfang Li, ${ }^{\ddagger}$ Puyu Yang, ${ }^{\ddagger}$ Jianchun Wan, ${ }^{\ddagger}$ Qimeng Chang, ${ }^{\S}$ Thomas T. Y. Wang, "Weiying Lu, \\ Yaqiong Zhang, ${ }^{\dagger}$ Qin Wang, ${ }^{\perp}$ and Liangli Lucy $\mathrm{Yu}^{*}{ }^{+}{ }^{(0)}$ \\ ${ }^{\dagger}$ Beijing Advanced Innovation Center for Food Nutrition and Human Health, Beijing Technology \& Business University (BTBU), \\ Beijing 100048, China \\ ${ }^{\ddagger}$ Institute of Food and Nutraceutical Science, School of Agriculture and Biology, Shanghai Jiao Tong University, Shanghai 200240, \\ China \\ ${ }^{\S}$ Department of Surgery, Fudan University Minhang Hospital, Shanghai 201199, China \\ "Diet, Genomics, and Immunology Laboratory, Agricultural Research Service (ARS), USDA, Beltsville, Maryland 20705, United \\ States \\ ${ }^{\perp}$ Department of Nutrition and Food Science, University of Maryland, College Park, Maryland 20742, United States
}

\section{Supporting Information}

ABSTRACT: This study investigated whether and how gypenosides from jiaogulan tea at 100 and $300 \mathrm{mg} / \mathrm{kg} / \mathrm{day}$ levels could reduce the development of overweight and insulin resistance in C57 BL/6J mice fed a high-fat diet in 12 weeks. The $300 \mathrm{mg} / \mathrm{kg} /$ day gypenosides supplement significantly reduced final body weight, plasma total cholesterol, and homeostasis model assessment-estimated insulin resistance (HOMA-IR) index by 19.9\%, 40\%, and 36\%, respectively, compared with the high-fat diet control group. Gypenosides also increased brown adipocyte tissue activity and white adipose tissue browning. The expression of genes involved in mitochondrial activity and fatty acid $\beta$-oxidation were also increased in both brown and white adipocyte tissues. In addition, gypenosides at 100 and $300 \mathrm{mg} / \mathrm{kg} /$ day levels decreased the ratio of Firmicutes to Bacteroidetes by $20 \%$ and 58.6\%, respectively, and increased Akkermansia muciniphila abundance in the gut microbiota.

KEYWORDS: gypenosides, obesity, insulin resistance, white adipocyte tissue, brown adipocyte tissue, microbiota

\section{INTRODUCTION}

White adipose tissue (WAT) plays an important role in regulating the whole body homeostasis and stores energy in the triglycerides (TGs) form, whereas brown adipose tissue (BAT) utilizes lipids for heat production. The BAT function is mediated by the tissue-specific uncoupling protein 1 (UCP-1) located in the mitochondria and dissociates cellular respiration from ATP utilization to convert the stored energy to heat. ${ }^{1,2}$ BAT activity in humans is inversely correlated with adiposity, blood glucose concentrations, and insulin resistance. ${ }^{2}$ Manipulating WAT to acquire BAT-like characteristics is considered a possible approach to reduce the risk of overweight and obesity. ${ }^{3}$ Despite advances in knowledge regarding the developmental lineage and transcriptional regulators of brown and beige adipocytes, current knowledge on whether and how food factors and other environmental modifiers may alter BAT thermogenesis is limited. 4

Jiaogulan, Gynostemma pentaphyllum Makino (GP), is a perennial liana vine widely spread in Asian countries, and its leaves have been used in food and tea. ${ }^{6}$ Gypenosides are considered the major bioactive constituents in GP and have been shown to reduce the potential development of obesity, ${ }^{7}$ cardiovascular diseases, ${ }^{8}$ diabetes, ${ }^{9}$ inflammation, ${ }^{6,10}$ and cancer. $^{11,12}$ A previous study showed that gypenosides could curb obesity in an $o b / o b$ mouse model through activating AMP- activated protein kinase. ${ }^{13}$ Park et al. confirmed the potential antiobesity and safety of gypenosides at $450 \mathrm{mg} / \mathrm{kg} /$ day level through a 12 -week, 80 Korean participant, randomized, doubleblind, placebo-controlled clinical trial. ${ }^{7}$ Interestingly, gypenosides were able to inhibit adipogenesis through modulating the $\mathrm{Wnt} / \beta$-catenin pathway and cell cycle in mitotic clonal expansion in one of our recent studies. ${ }^{14}$ However, these previous studies did not investigate the possible molecular mechanisms behind the antiobesity capacity of gypenosides or their effects on adipocytes such as stimulating BAT activity and browning of white adipose tissue.

In addition, gut microbiota may play a role in the development of the obese and diabetic phenotypes. ${ }^{15}$ An increase in the major phyla Firmicutes/Bacteroidetes ratio and changes in several bacterial species have been shown to increase the risk of obesity in both genetic and dietary model mice. ${ }^{16}$ Alterations in the gut microbial community may impact the host capacity to extract energy from a given diet, thereby triggering the development of obesity and related diabetes. ${ }^{17,18}$ Another study reported that transplanting the "cold microbiota"

Received: July 21, 2017

Revised: October 3, 2017

Accepted: October 4, 2017

Published: October 4, 2017 
to germ-free mice was able to increase insulin sensitivity of the host and its tolerance to cold partially through inducing the white fat browning and resulting in an increased fat loss and energy expenditure. ${ }^{1}$ The cold microbiota referred to the microbiota in $\mathrm{C} 57 \mathrm{BL} / 6$ mice exposed to $6{ }^{\circ} \mathrm{C}$ for 30 days under a SPF condition. The results indicate a possible link between the gut microbiota and the energy homeostasis of the host. ${ }^{1}$ Therefore, it is interesting whether gypenosides may alter glucose metabolism and body weight gain through modulating the gut microbiota.

In this study, the effect of gypenosides on the risk of obesity and insulin resistance and their possible roles in $\beta$-oxidation and mitochondrial function, BAT activity, and WAT browning were investigated in a high-fat diet-induced obese mouse model. Their effects on gut microbiota composition were also examined as a possible mechanism behind their capacity in reducing the development of overweight and related diabetes.

\section{MATERIALS AND METHODS}

Reagents and Materials. The leaves of tetraploid jiaogulan (G. pentaphyllum (Thunb.) Makino) were collected and identified by Baicaotang Biotechnology Co. Ltd., Pingli, Shanxi Province of China, in 2013. Botanicals were kept at $4{ }^{\circ} \mathrm{C}$ until extraction. A voucher specimen (no. 2013003) has been deposited in our laboratory, at the School of Agriculture and Biology, Shanghai Jiao Tong University, Shanghai, China. Gypenosides were prepared according to a laboratory procedure, which contained nine different dammarane-type saponins with a purity $>95 \%$.

Gypenosides contained 10.84\% (GP-1), 7.57\% (GP-2), 24.10\% (GP 3 + GP 4), 26.12\% (GP 5 + GP 6 + GP 7), 24.76\% (GP-8), and 5.60\% (GP-9). ${ }^{19}$

Animal and Diet. Thirty-two male C57 BL/6J mice (5 weeks old, Charles River Laboratories, Beijing, China) were used in this study. All animal experiment procedures were approved with permission number A2015024 by the Medical Ethics Committee of Shanghai Jiao Tong University and in accordance with the National Institutes of Health regulations for the care and use of animals in research. All animals were housed individually in polycarbonate cages in an animal care facility at $22-24{ }^{\circ} \mathrm{C}$ and $40-50 \%$ relative humidity, lights were on during 9:00-21:00, and mice were fed the AIN-76 semipurified diet for a 1-week acclimation period. They were subsequently divided randomly into four groups and fed a low-fat diet (LFD, $n=8$, D12450B, Research Diets Inc., NJ, USA), high-fat diet (HFD, $n=8$, D12451, Research Diets Inc., NJ, USA), or HFD with $100 \mathrm{mg} / \mathrm{kg}$ gypenosides ( $\mathrm{HFD}+\mathrm{L}, n=8)$ and $300 \mathrm{mg} / \mathrm{kg}$ gypenosides $(\mathrm{HFD}+\mathrm{H}, n$ $=8$ ) for 12 weeks. All experimental diets were changed every 3 days and stored at $4{ }^{\circ} \mathrm{C}$. The body weights and food intakes were monitored every week. At the end of the experimental period, all mice were anesthetized after a $12 \mathrm{~h}$ fast. Blood was taken from the inferior vena cava to determine the glucose, plasma lipid, and insulin concentrations. Feces were collected for three consecutive days prior to animal sacrifice and stored at $-80{ }^{\circ} \mathrm{C}$ for macrobiotic analysis. All organ tissues were harvested, weighed, immediately frozen in RNALater (Invitrogen) or liquid nitrogen, and stored at $-80^{\circ} \mathrm{C}$ for biochemical and molecular analyses.

Oral Glucose Tolerance Test (OGTT) and Intraperitoneal Insulin Tolerance Test (IPITT). After 12 weeks of different concentrations of gypenosides administration, the mice were fasted 6 $\mathrm{h}$ and orally given glucose at a $1.5 \mathrm{~g} / \mathrm{kg}$ body weight level for oral glucose tolerance test (OGTT) and fasted for $4 \mathrm{~h}$ and intraperitoneally injected insulin at a concentration of $0.75 \mathrm{unit} / \mathrm{kg}$ body weight for intraperitoneal insulin tolerance test (IPITT). The blood glucose concentrations were determined at $0,30,60,90$, and $120 \mathrm{~min}$ after feeding or injection with a portable glucose meter (One-touch UltraEasy, Johnson Medical, Beijing, China). A glucose concentration-time plot was prepared to show the changes in glucose levels with time and to calculate the integrated areas under each curve (AUC) for OGTT and IPITT. ${ }^{20}$
Quantification of Plasma Lipids, Glucose, and Homeostatic Model Assessment of Insulin Resistance (HOMA-IR). Plasma samples were obtained by centrifugation at $3500 \mathrm{rpm}$ and $4{ }^{\circ} \mathrm{C}$ for 10 min. Plasma total cholesterol (TC) and triglycerides (TG) were enzymatically determined using commercial kits (LabAssay Triglyceride/Cholesterol Kit, Wako, Osaka, Japan), and fasting glucose concentrations were determined using the portable glucose meter. The plasma insulin levels were measured by ELISA assays according to the manufacturer's instructions (EZRMI-13K, Millipore, Germany). HOMA-IR index was calculated as [fasting insulin concentration $(\mathrm{mU} / \mathrm{L}) \times$ fasting glucose concentration $(\mathrm{mmol} / \mathrm{L})] / 22.5 .^{20}$

Morphological and Immunohistochemistry. Perirenal adipose tissues were fixed in $4 \%$ paraformaldehyde and were sectioned after being paraffin embedded. Multiple sections were stained with hematoxylin and eosin for general morphological observations. Immunohistochemistry staining was performed according to the standard protocol using the UCP1 antibody with 1:100 dilution (Abcam, Oxford, UK).

Real-Time PCR. To measure mRNA expression changes, different adipose tissues with different treatments preserved in RNALater were cut into $0.1-0.2 \mathrm{~g}$ pieces and homogenized using a Precellys 24 (Bertin Technologies, Villeurbanne, France). RNeasy Mini Kit (Qiagen, Valencia, CA) was used for total RNA isolation. A $0.2 \mu \mathrm{g}$ amount of first-strand cDNA synthesis was performed using an iScript reverse transcriptase kit (Bio-Rad) and subsequently used for quantitative real-time PCR analysis. qRT-RCR was conducted using SYBR Green mix and ABI 7900 HT (Applied Biosystems, Carlsbad, CA). PCR amplification was performed as follows: $50^{\circ} \mathrm{C}$ for $2 \mathrm{~min}, 95$ ${ }^{\circ} \mathrm{C}$ for $10 \mathrm{~min}$, and 46 cycles of amplification at $95{ }^{\circ} \mathrm{C}$ for $15 \mathrm{~s}$ and 60 ${ }^{\circ} \mathrm{C}$ for $1 \mathrm{~min}$. Statistical analysis of quantitative real-time PCR was performed using the $2^{-\Delta \Delta C t}$ method as previously reported, ${ }^{19}$ which calculates the relative changes in gene expression of the target, normalized to the endogenous reference $\beta$-actin and relative to a control calibrator. Forward and reverse primer sequences are shown in Table S1 in the Supporting Information.

Western Blotting. Homogenized tissue samples were lysed in 200 $\mu \mathrm{L}$ of ice-cold radioimmunoprecipitation assay (RIPA) buffer containing a protease inhibitor cocktail (Sigma, St. Louis, MO, USA) and phosphatase inhibitors (Roche Diagnostics, Mannheim, Germany). To remove the insoluble materials, lysates were centrifuged at $10000 \mathrm{~g}$ for $20 \mathrm{~min}$ at $4{ }^{\circ} \mathrm{C}$. The protein concentrations in the lysates were examined using a BCA protein assay kit (Pierce, Rockford, IL, USA) according to the manufacturer protocol. The protein concentration for each sample was adjusted to an equal amount with different volumes of loading buffer and denatured in boiling water for $5 \mathrm{~min}$. To separate different proteins, equal aliquots $(40 \mu \mathrm{g})$ of protein samples were subjected to $12 \%$ polyacrylamide gel electrophoresis and then electrotransferred to PVDF membranes at $25 \mathrm{~V}$ and $1.5 \mathrm{~A}$ for 60 min. The membranes were blocked with $5 \%$ nonfat milk in Trisbuffered saline containing $0.1 \%$ Tween-20 (TBST) for $2 \mathrm{~h}$. After being washed with TBST three times, the membranes were incubated with target protein-specific antibodies overnight at $4{ }^{\circ} \mathrm{C}$, followed by incubating with secondary antibodies with conjugated horseradish peroxidase (HRP) at ambient temperature for $1.5 \mathrm{~h}$. Peroxidase activity was visualized using the chemiluminescence method with an ECL kit (Bio-Rad, Hercules, CA, USA) with ChemiDoc $\mathrm{XRS}^{+}$(BioRad). $\beta$-Actin and $\alpha$-tubulin were used as internal controls.

Fecal DNA Extraction and Amplification. Four mice from the 8 mice in each diet group were randomly selected for fecal microbiome analyses. The QIAamp DNA Mini Kit (Qiagen, Valencia, CA, USA) was used to isolate total bacterial DNA from the frozen fecal samples according to the manufacturer's instructions. The extracted DNA was measured for concentrations using a Nano-Drop 1000 spectrophotometer (Nano-Drop Technologies, Wilmington, DE). The extracted DNA samples were stored at $-80{ }^{\circ} \mathrm{C}$ for further analysis. To examine the composition of the microbiota community, universal forward primers $338 \mathrm{~F}$ (5'-ACTCCTACGGGAGGCAGCA-3') and the reverse primer $806 \mathrm{R}$ (5'-GGACTACHVG GGTWTCTAAT-3') targeting the V3-V4 region of the $16 \mathrm{~S}$ rRNA gene were utilized for the amplification and sequencing. The targeted 16S rRNA gene was 
Table 1. Food Intake, Body Weight, Organ Weights, and Biochemical Parameters ${ }^{a}$

\begin{tabular}{|c|c|c|c|c|}
\hline & LFD & HFD & $\mathrm{HFD}+\mathrm{L}$ & $\mathrm{HFD}+\mathrm{H}$ \\
\hline food intake (g/day) & $3.44 \pm 0.33 a$ & $3.65 \pm 0.52 \mathrm{a}$ & $3.54 \pm 0.32 \mathrm{a}$ & $3.63 \pm 0.42 \mathrm{a}$ \\
\hline energy intake (kcal/day) & $13.24 \pm 1.27 \mathrm{a}$ & $17.26 \pm 2.45 b$ & $16.74 \pm 1.51 \mathrm{~b}$ & $17.16 \pm 1.99 b$ \\
\hline $\begin{array}{l}\text { final body weight }(\mathrm{g}) \\
\text { organ weights }(\mathrm{g})\end{array}$ & $28.08 \pm 0.98 a$ & $35.42 \pm 1.74 b$ & $31.58 \pm 3.18 \mathrm{ab}$ & $28.37 \pm 2.30 \mathrm{a}$ \\
\hline liver & $0.85 \pm 0.07 a$ & $1.20 \pm 0.11 c$ & $1.01 \pm 0.08 \mathrm{~b}$ & $0.93 \pm 0.11 \mathrm{ab}$ \\
\hline kidney & $0.33 \pm 0.04 a$ & $0.41 \pm 0.04 b$ & $0.37 \pm 0.04 b$ & $0.38 \pm 0.02 b$ \\
\hline testes & $0.19 \pm 0.03 a$ & $0.18 \pm 0.08 \mathrm{a}$ & $0.18 \pm 0.03 a$ & $0.20 \pm 0.02 \mathrm{a}$ \\
\hline lung & $0.16 \pm 0.02 \mathrm{a}$ & $0.18 \pm 0.02 \mathrm{a}$ & $0.17 \pm 0.02 \mathrm{a}$ & $0.19 \pm 0.07 \mathrm{a}$ \\
\hline brain & $0.30 \pm 0.01 \mathrm{a}$ & $0.38 \pm 0.01 b$ & $0.38 \pm 0.06 b$ & $0.39 \pm 0.03 b$ \\
\hline spleen & $0.06 \pm 0.01 \mathrm{a}$ & $0.07 \pm 0.02 \mathrm{a}$ & $0.07 \pm 0.01 \mathrm{a}$ & $0.06 \pm 0.01 \mathrm{a}$ \\
\hline pancreas & $0.11 \pm 0.01 \mathrm{a}$ & $0.12 \pm 0.02 \mathrm{a}$ & $0.14 \pm 0.06 a$ & $0.13 \pm 0.04 \mathrm{a}$ \\
\hline thymus & $0.04 \pm 0.03 a$ & $0.05 \pm 0.00 \mathrm{a}$ & $0.04 \pm 0.02 \mathrm{a}$ & $0.03 \pm 0.02 \mathrm{a}$ \\
\hline epididymat fat & $0.38 \pm 0.09 a$ & $1.43 \pm 0.14 b$ & $1.11 \pm 0.40 \mathrm{~b}$ & $0.60 \pm 0.31 \mathrm{a}$ \\
\hline subcutaneous fat & $0.28 \pm 0.05 a$ & $0.92 \pm 0.14 c$ & $0.79 \pm 0.25 b c$ & $0.50 \pm 0.22 \mathrm{ab}$ \\
\hline $\begin{array}{l}\text { inguinal fat } \\
\text { plasma parameters }\end{array}$ & $0.08 \pm 0.03 a$ & $0.56 \pm 0.06 c$ & $0.41 \pm 0.19 b c$ & $0.30 \pm 0.20 \mathrm{ab}$ \\
\hline $\mathrm{TC}(\mathrm{mg} / \mathrm{dL})$ & $131.74 \pm 15.45 a$ & $193.67 \pm 22.81 b$ & $179.36 \pm 36.52 \mathrm{ab}$ & $125.97 \pm 13.5 a$ \\
\hline $\mathrm{TG}(\mathrm{mg} / \mathrm{dL})$ & $264.13 \pm 43.47 a$ & $266.79 \pm 31.32 \mathrm{a}$ & $257.29 \pm 25.32 \mathrm{a}$ & $259.25 \pm 53.55 a$ \\
\hline fasting glucose $(\mathrm{mmol} / \mathrm{dL})$ & $11.43 \pm 1.55 \mathrm{a}$ & $12.07 \pm 1.66 \mathrm{a}$ & $11.30 \pm 1.68 \mathrm{a}$ & $11.50 \pm 2.73 a$ \\
\hline insulin $(\mathrm{ng} / \mathrm{mL})$ & $0.50 \pm 0.05 a$ & $0.82 \pm 0.11 b$ & $0.67 \pm 0.12 \mathrm{ab}$ & $0.55 \pm 0.10 \mathrm{a}$ \\
\hline HOMA-IR & $6.27 \pm 1.02 \mathrm{a}$ & $10.87 \pm 1.25 b$ & $8.31 \pm 1.45 \mathrm{ab}$ & $6.96 \pm 0.89 a$ \\
\hline
\end{tabular}

${ }^{a}$ LFD represents mice fed a low-fat diet, HFD represents mice fed a high-fat diet, and HFD+L and HFD+H represent mice fed HFD plus 100 and $300 \mathrm{mg} / \mathrm{kg}$ gypenosides, respectively. Values are means $\pm \mathrm{SD}(n=8)$; different letters indicate a significant difference of data in a row at $P<0.05$.

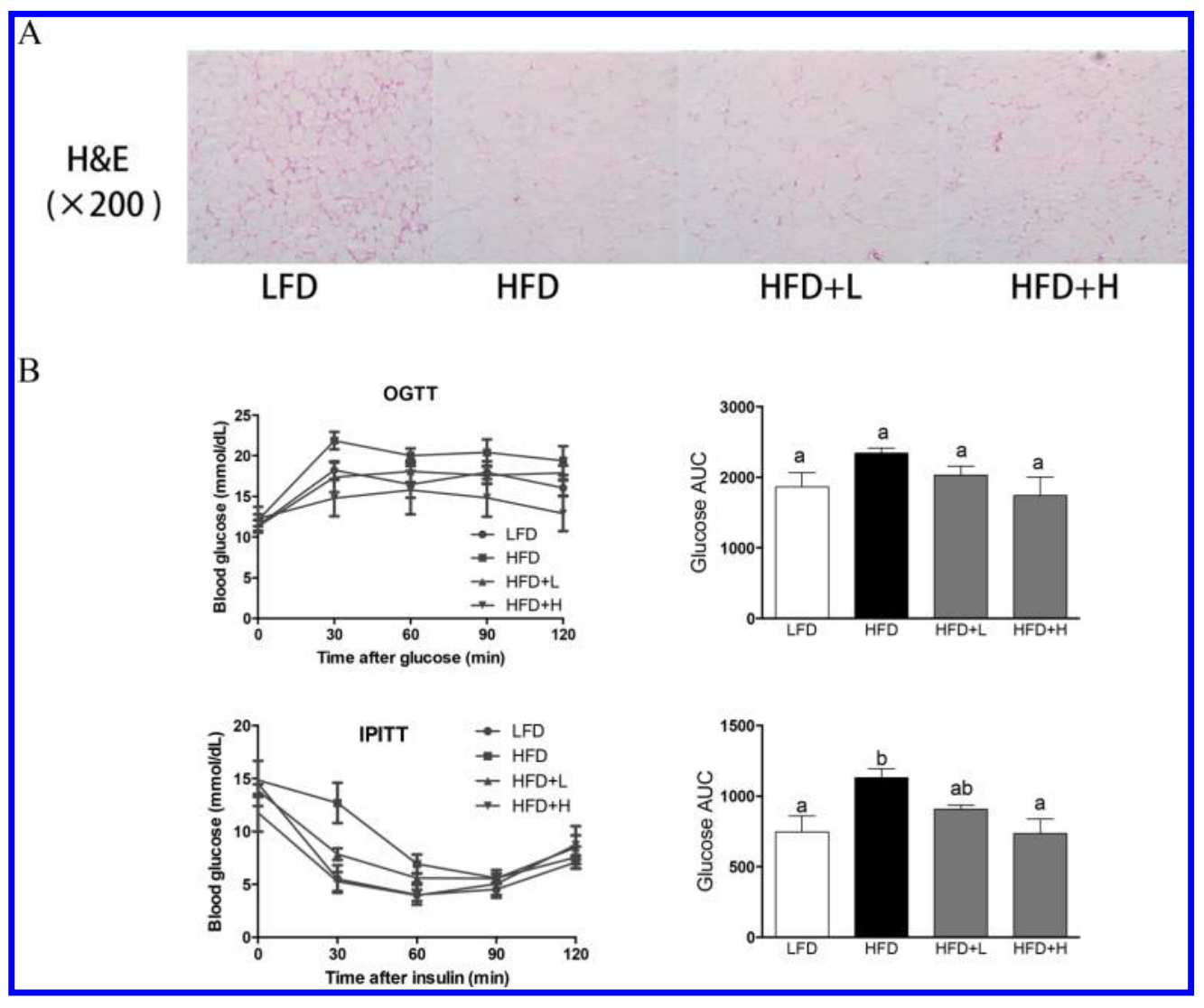

Figure 1. Effect of gypenosides on hematoxylin and eosin (H\&E) staining of inguinal white adipocyte tissue (iWAT) (A) and oral glucose tolerance test (OGTT) and intraperitoneal insulin tolerance test (IPITT) (B). LFD represents mice fed a low-fat diet, HFD represents mice fed a high-fat diet, and HFD $+\mathrm{L}$ and HFD+H represent mice fed HFD plus 100 and $300 \mathrm{mg} / \mathrm{kg}$ gypenosides, respectively. Values are means \pm SD ( $n=8)$. Values marked with different letters are significantly different from each other at $P<0.05$.

PCR amplified in triplicate. PCR conditions were a denaturing step at $95{ }^{\circ} \mathrm{C}$ for $3 \mathrm{~min}$ and 27 cycles of denaturing at $95{ }^{\circ} \mathrm{C}$ for $30 \mathrm{~s}$, annealing at $55{ }^{\circ} \mathrm{C}$ for $30 \mathrm{~s}$, and extension at $72{ }^{\circ} \mathrm{C}$ for $45 \mathrm{~s}$, followed by a final annealing extension step at $72{ }^{\circ} \mathrm{C}$ for $10 \mathrm{~min}$. PCR products 


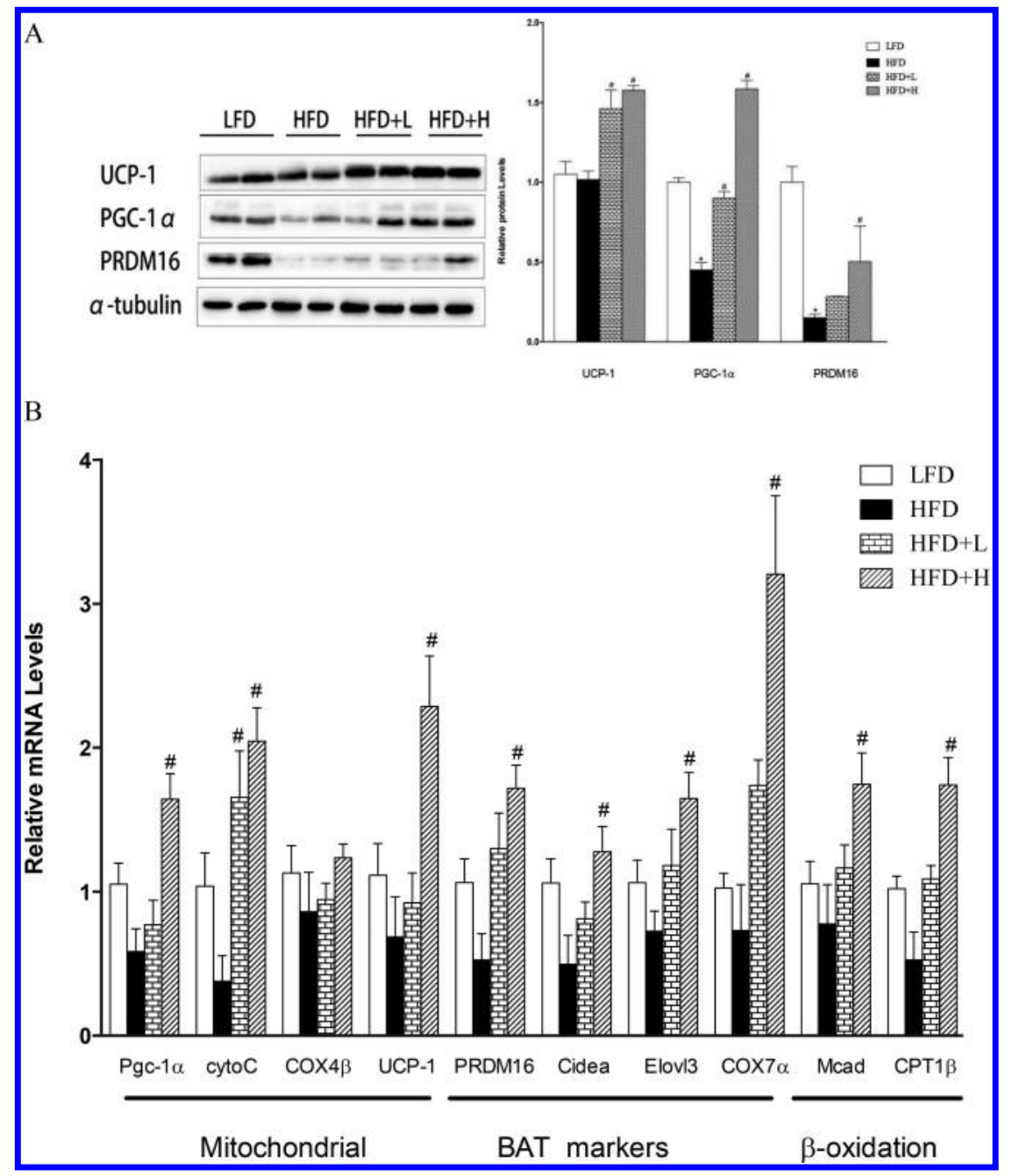

Figure 2. Effect of gypenosides on genes expression in brown adipocyte tissue (BAT) at both protein (A) and mRNA (B) levels. LFD represents mice fed a low-fat diet, HFD represents mice fed a high-fat diet, and HFD+L and HFD+H represent mice fed HFD plus 100 and $300 \mathrm{mg} / \mathrm{kg}$ gypenosides, respectively. Values are means \pm SD $(n=8):(*)$ significant difference between HFD and LFD groups $(P<0.05),(\#)$ significant difference between the gypenosides treatment group and the HFD group $(P<0.05)$.

were visualized, purified using a Mini Elute PCR purification kit (AXYGEN), and quantified using an ABI GeneAmp1 9700 system. Sequencing was accomplished on the Illumina MiSeq platform at Shanghai Majorbio Biopharm Technology Co., Ltd. (Shanghai, China). The 16S rDNA sequences were deposited with the NCBI short reads archive database with a study accession number of PRJNA303100.

Bioinformatics Analysis and Statistical Analyses. The processing and bioinformatics analysis of the sequencing data were similar to that described in the previous study. ${ }^{21}$ Raw fastq files were demultiplexed and quality-filtered using QIIME (version 1.9.1) under the following conditions: (1) the $300 \mathrm{bp}$ reads were truncated at any site receiving an average quality score $<20$ over a 50 bp sliding window, while the truncated reads shorter than 50 bp were discarded; (2) exact barcode matching, 2 nucleotide mismatch in primer matching and the reads having ambiguous characters were discarded, and only sequences overlapping for over $10 \mathrm{bp}$ were assembled according to their overlap sequences; (3) the reads were discarded if they could not be assembled. ${ }^{21}$

A 97\% similarity cutoff was applied to cluster operational taxonomic units (OTUs) with Usearch (version 7.1, http://drive5.com/uparse/), and identified chimeric sequences were removed with UCHIME. The taxonomy of each 16SrRNA gene sequence was examined using RDP Classifier (http://rdp.cme.msu.edu/) against the silva (SSU123) $16 \mathrm{~S}$ rRNA database, and a confidence threshold was set at $70 \%$. The linear discriminant analysis effect size (LEfSe) method (http://huttenhower. sph.harvard.edu/lefse/) was utilized on OUT table to identify biomarkers. LEfSe analysis was performed with the alpha value of 0.05 for the factorial Kruskal-Wallis test among classes and the threshold of 4.0 on the logarithmic LDA score for discriminative features.

Data were expressed as mean $\pm \mathrm{SD}$. The statistical significance of the difference was analyzed using one-way ANOVA, followed by Tukey's multiple comparison test. All statistical analyses were performed with SPSS 21.0 software. $P<0.05$ was considered to be statistically significant. The graph was created on GraphPad Prism (Version 6.00, Graphpad Software Inc., San Diego, CA, USA).

\section{RESULTS AND DISCUSSION}

Gypenosides Reduced HFD-Induced Body Weight and Insulin Resistance. Gypenosides dose-dependently decreased final body weight and fat accumulation in a highfat diet (HFD)-induced obese mouse model (Table 1). Histological analysis for inguinal white adipose tissue suggested that HFD promoted adipocyte hypertrophy with a significant increase of adipocyte area compared to the LFD group, and this was prevented by gypenosides supplementation in a dosedependent manner (Figure 1A). The effects of gypenosides on body weight and obesity parameters were not a result of a reduced food consumption as there was no difference in the 


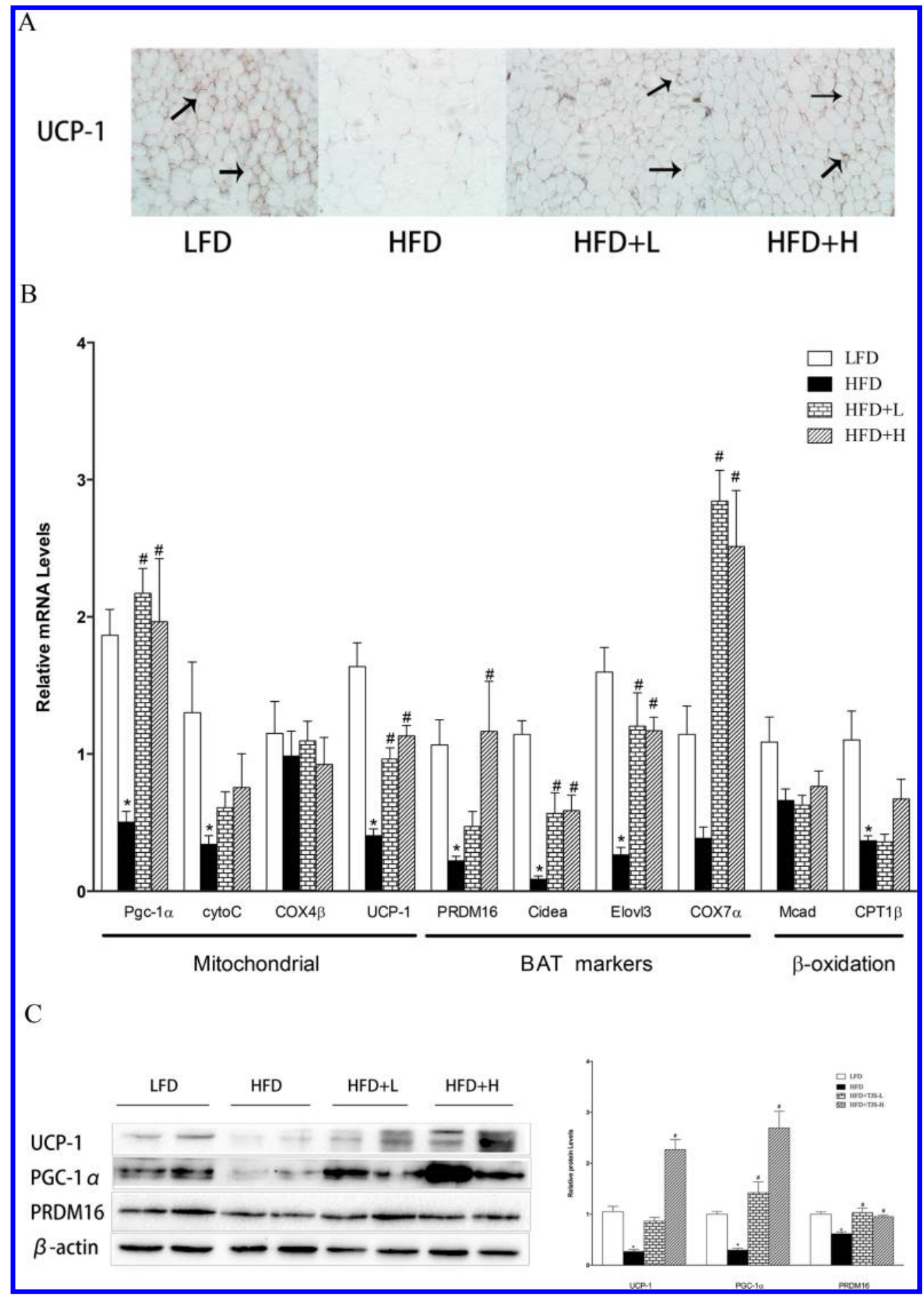

Figure 3. Effect of gypenosides on immunohistochemistry of UCP-1 (A), and genes expression in inguinal white adipocyte tissue (iWAT) at both mRNA (B) and protein (C) levels. LFD represents mice fed a low-fat diet, HFD represents mice fed a high-fat diet, and HFD+L (HFD+TJS-L) and $\mathrm{HFD}+\mathrm{H}(\mathrm{HFD}+\mathrm{TJS}-\mathrm{H})$ represent mice fed HFD plus 100 and $300 \mathrm{mg} / \mathrm{kg}$ gypenosides, respectively. Values are means \pm SD $(n=8):(*)$ significant difference between HFD and LFD groups $(P<0.05)$; $(\#)$ significant difference between the gypenosides treatment group $(s)$ and the HFD group $(P$ $<0.05)$.

food intakes among the treatment groups (Table 1). A $300 \mathrm{mg}$ / $\mathrm{kg}$ /day gypenosides treatment with HFD also significantly reduced epididymal, subcutaneous, and inguinal fats by $58 \%$, $45.7 \%$, and $46.4 \%$, respectively, compared with the HFD group $(P<0.05)$, suggesting their potential in inhibiting fat accumulation (Table 1). Moreover, no significant differences were observed on the fasting blood glucose (Table 1) and in the oral glucose tolerance test (OGTT) among the treatment groups (Figure 1B). The $\mathrm{AUC}_{\text {glucose }}$ for the OGTT reflects the degree of glucose tolerance in the mice, and no significant difference among treatment groups (Figure 1B) indicated no glucose intolerance under the experimental conditions. Interestingly, treatment with $300 \mathrm{mg} / \mathrm{kg}$ gypenosides significantly reduced the plasma insulin concentration and the 


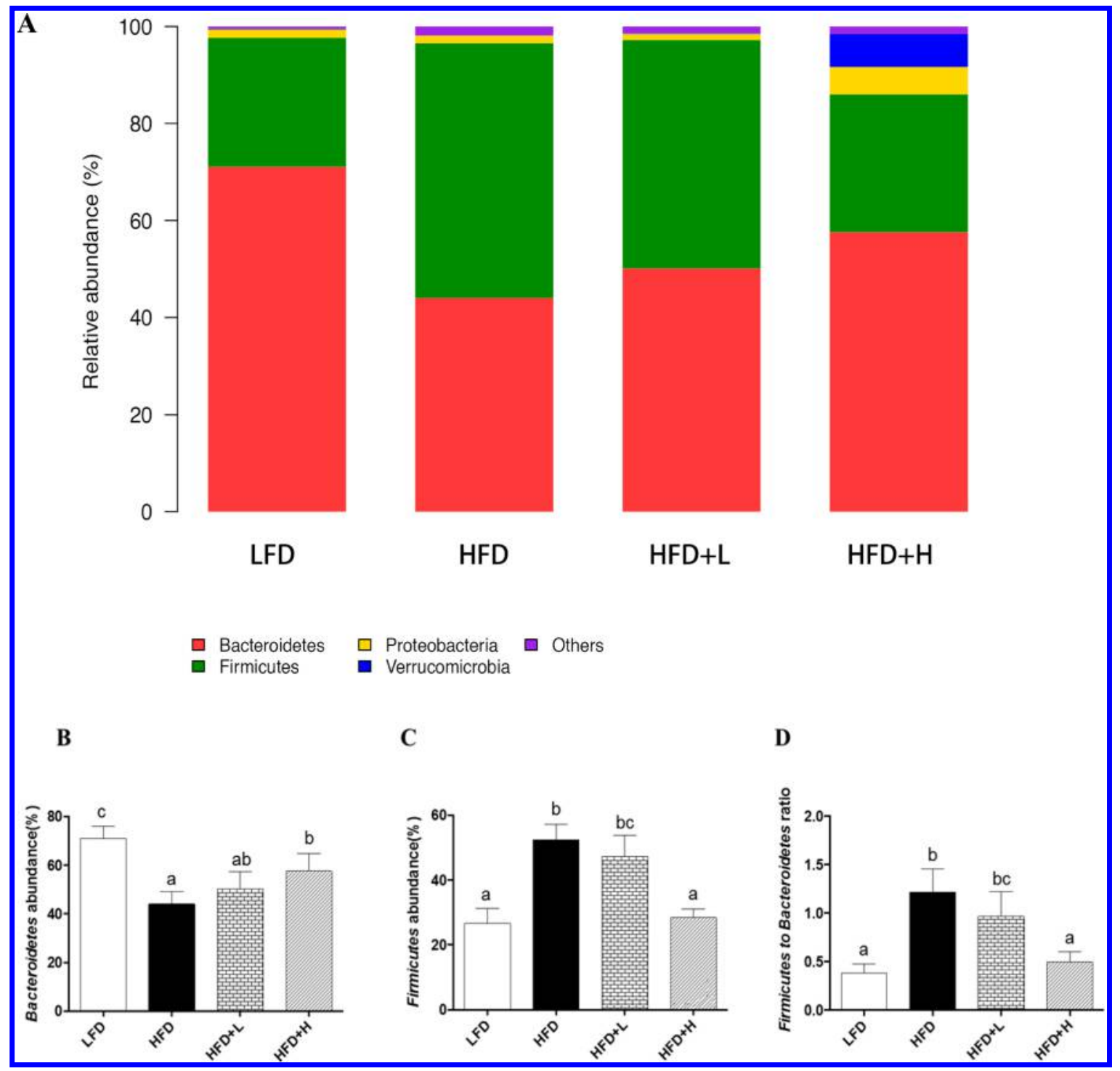

Figure 4. Fecal bacterial community at the phylum level (A). Relative abundance of different bacterial phylum within the different communities (BD). LFD represents mice fed a low-fat diet, HFD represents mice fed a high-fat diet, and HFD $+\mathrm{L}$ and HFD $+\mathrm{H}$ represent mice fed HFD plus 100 and $300 \mathrm{mg} / \mathrm{kg}$ gypenosides, respectively. Values are means $\pm \mathrm{SD}(n=4)$. Values marked with different letters are significantly different from each other at $P<0.05$.

HOMA-IR by $14.9 \%$ and $36 \%$, respectively, compared with that of the high-fat diet group $(P<0.05)$, indicating a possible reduction in obese-associated insulin resistance (Table 1 ). The improvement of insulin resistance was also supported by the intraperitoneal insulin tolerance test (IPITT) result (Figure 1B). After intraperitoneal injection of insulin, the plasma glucose levels of mice in the HFD group decreased significantly less than the other three diet groups at $30 \mathrm{~min}$, but the four groups had no difference in their plasma glucose levels at 60, 90, and $120 \mathrm{~min}$ (Figure $1 \mathrm{~B}$ ), indicating the potential of gypenosides in improving insulin sensitivity in the HFD mice.

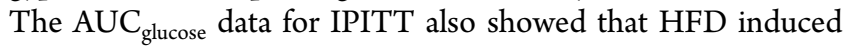
insulin intolerance in mice, and gypenosides treatment was able to reverse the insulin intolerance in a dose-dependent matter. These results implied that gypenosides could reduce body weight, fat accumulation, and overweight-induced insulin resistance.

Gypenosides-Induced Thermogenesis of BAT and Inguinal WAT (iWAT). The effect of gypenosides on the selected BAT thermogenic progress marker proteins including PPAR- $\gamma$ coactivator- $1 \alpha$ (PGC- $1 \alpha$ ), PR domain containing 16 (PRDM16), and UCP1 were investigated using a western-blot analysis, and two representative SDS-PAGE results from eight animals in each dietary treatment group are reported in Figure 2A. Compared with the HFD mice, supplementation with gypenosides significantly increased UCP-1, PGC- $1 \alpha$, and PRDM16 protein expressions in a dose-dependent manner $(P$ $<0.05$, Figure 2A). UCP1 is important for BATs in transforming stored chemical energy into heat, a thermal energy, and protecting mice from overweight and cold, ${ }^{4}$ whereas increased PGC- $1 \alpha$ and PRDM16 are associated with adipocyte tissue energy expenditure. Moreover, gypenosides were able to significantly promote the mRNA expressions of mitochondrial activity and lipid $\beta$-oxidation genes, including $P G C-1 \alpha, P R D M 16$, cell death-inducing DNA fragmentation factor $(\operatorname{Cide} \alpha)$, cytochrome $c$ oxidase, subunit VII $\alpha(\operatorname{Cox} 7 \alpha)$, long-chain fatty acids-like 3 (Elovl3), carnitine palmitoyl transterase-1 (CPT1), and medium-chain acyl-CoA dehydro- 


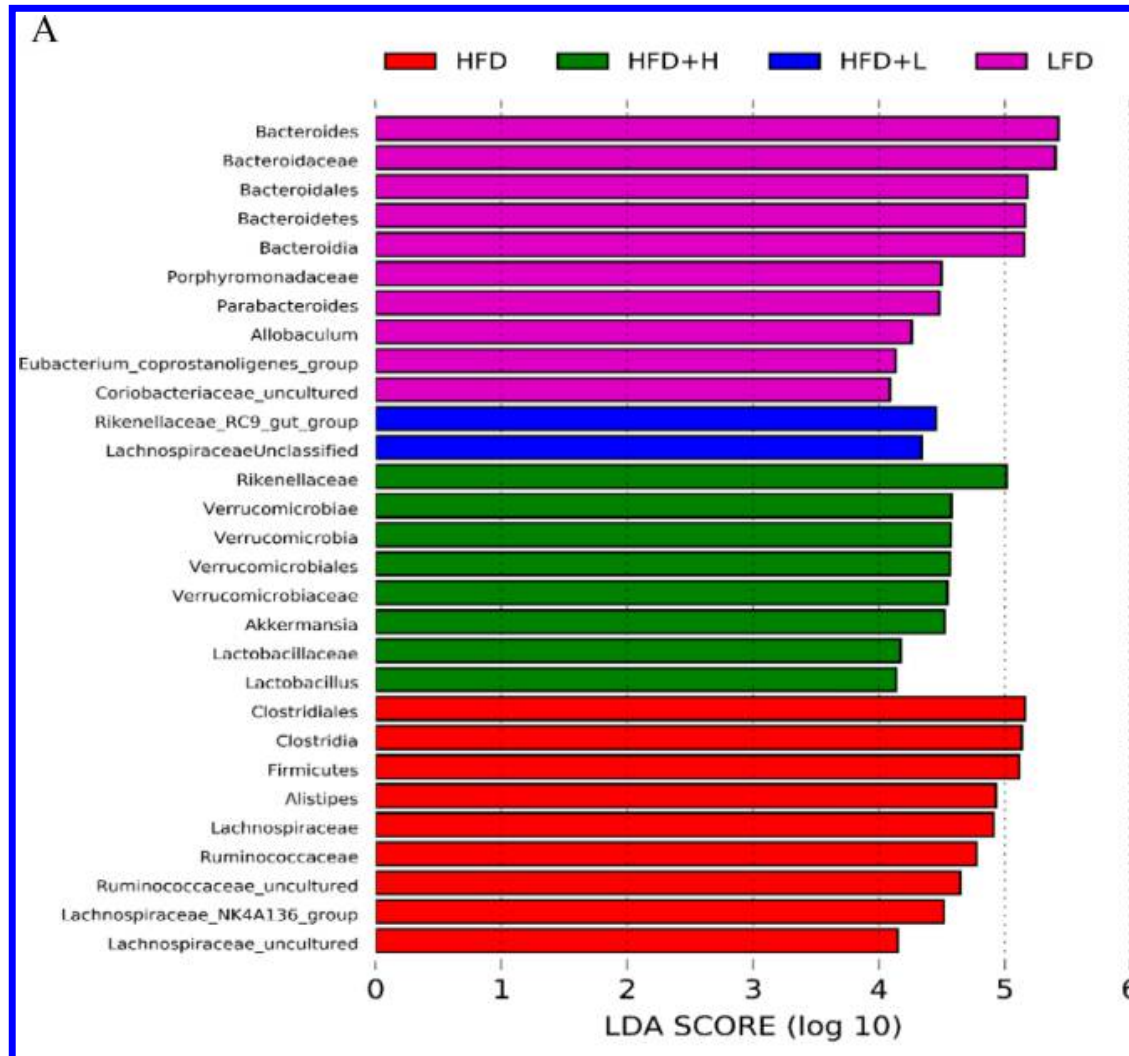

B

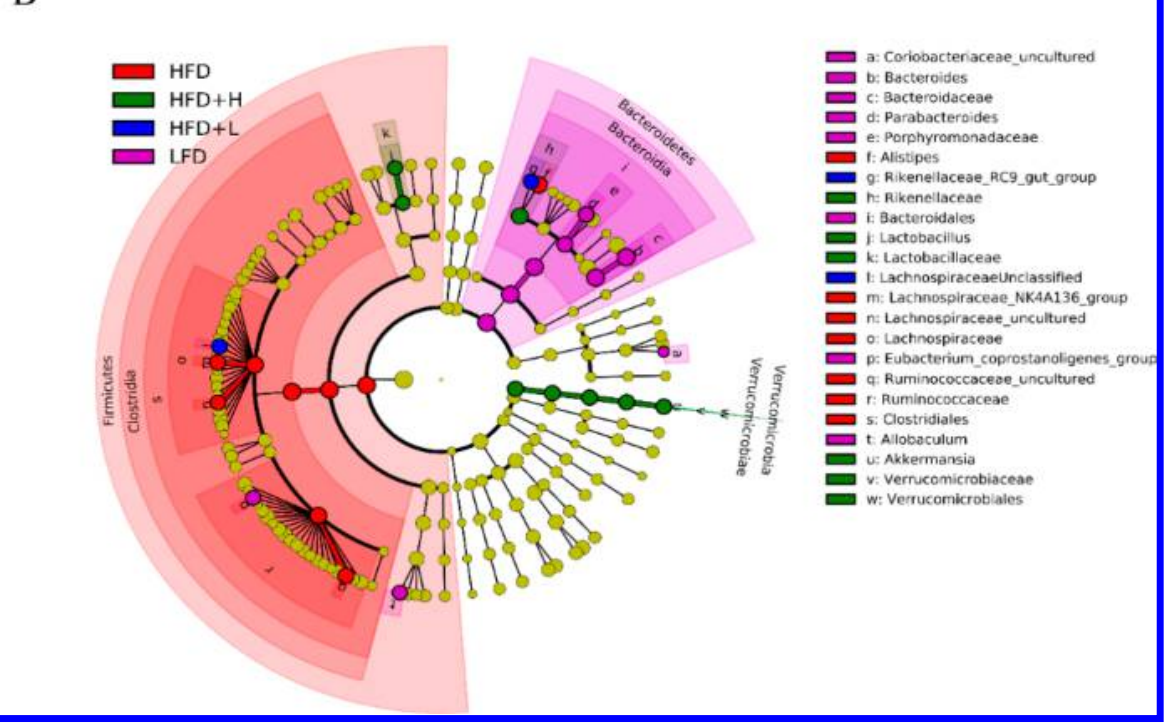

Figure 5. LEfSe analysis of key genera of mice gut microbiota. Histogram shows the LDA scores computed for features (OTU level) differentially abundant between different treatments. The higher the score is, the more important the role is (A). Cladogram shows that brown dots are unimportant bacteria in any groups; other colored dots are important bacteria in the group labeled with the same color (B). LFD represents mice fed a low-fat diet, HFD represents mice fed a high-fat diet, and HFD+L and HFD+H represent mice fed HFD plus 100 and $300 \mathrm{mg} / \mathrm{kg}$ gypenosides, respectively.

genase $(\mathrm{MCAD})(P<0.05$, Figure $2 \mathrm{~B})$ in a dose-dependent manner in the HFD-induced obese mouse model.

It has been reported that beige adipocytes from WATs are also UCP1 positive and may be induced to express thermogenic genes and stimulate respiration and energy expenditure besides the classical brown adipocytes from BATs, and the progress has been described as browning. ${ }^{22}$ Gypenosides was further evaluated for their potential on thermogenic program in WATs. Inguinal adipose tissue (iWAT) was used for UCP1 immunohistochemistry, and the results indicated that gypenosides treatment resulted in much denser small white adipocytes with clusters of UCP1 expressing in the multilocular adipocytes (Figure 3A). Meanwhile, gene expressions of the important markers of mitochondrial activity and brown adipocyte function, including PGC-1 $\alpha$, PRDM16, UCP-1, Cide $\alpha$, $\operatorname{Cox} 7 \alpha$, and Elovl3, were significantly increased by gypenosides treatment compared with the HFD control group $(P<0.05$, Figure 3B). Increased expression of key brown adipocyte 
marker proteins including PGC-1 $\alpha$, PRDM16, and UCP1 was also confirmed by a Western blot analysis (Figure 3C). These data indicated that gypenosides could increase the BAT thermogenic progress and induce adipocyte browning in iWAT.

Recently, increasing energy expenditure through nonshivering thermogenesis is considered a possible treatment of metabolic syndromes. ${ }^{3,23}$ Promoting energy expenditure through BAT thermogenesis or browning of iWAT is of significant interest as a potential antiobesity approach due to BAT and beige fat's ability to metabolize vast amounts of glucose and lipids in proportion to their tissue mass. ${ }^{23,24}$ In this study, dietary gypenosides showed potential in preventing highfat diet-induced overweight and insulin resistance through thermogenesis by activating PGC- $1 \alpha$ and PRDM16 genes expression. It has been shown that ectopic expression of PGC$1 \alpha$, a master regulator of mitochondrial biogenesis and function, in white adipocytes induced acquisition of BAT features, such as thermogenic markers expression. ${ }^{25}$ For example, dietary luteolin induced thermogenesis and browning in differentiated primary adipocytes through upregulating AMPK/PGC1 $\alpha$ pathway. It was also reported that PRDM16 was critical in the development and function of brown and beige adipocytes, and both beige adipocytes and PRDM16 were required for the "browning" of white fat tissues. ${ }^{26}$ The observations in this study were consistent with previous studies that PGC1 $\alpha$ and PRDM16 could be the key genes for thermogenesis effect of gypenosides. Besides, Broeders et al. showed that chenodeoxycholic acid (CDCA), a bile acid, promoted mitochondrial uncoupling via TGR5 in brown adipocytes and increased brown fat activity and energy expenditure in women. ${ }^{27}$ Bile acids were thought to increase BAT activity and enhance energy expenditure in murine BAT and human skeletal muscles through activating the TGR5 G protein-coupled receptor. ${ }^{27,28}$ Considering our previous study that gypenosides could increase bile acids excretion, ${ }^{19}$ it is interesting to further investigate whether TGR5 may be another possible gene involved in the brown adipocyte function and white adipocyte browning effects of gypenosides.

Gypenosides Reversed HFD-Induced Gut Dysbiosis. The gut microbiota is highly associated with development of obesity and type II diabetes. Feeding a HFD resulted in microbiota dysbiosis and increased fasting glucose levels and insulin intolerance. ${ }^{29}$ The effect of gypenosides on gut microbiota composition in mice was examined by performing a sequencing-based analysis of bacterial 16S rRNA (V3-V4 region) in cecal faeces. A data set of 896327 high-quality 16S rDNA gene sequences was obtained from 16 samples through MiSeq sequencing analysis, and the range in the number of reads for 16 samples was 31896-63986, while the average number of sequence reads was 47826 for each sample. The sequences were clustered with representative ones with a sequence identity cutoff of $97 \%$. At the phylum level, 11 phyla were identified in the faeces (Figure 4A). Firmicutes and Bacteroidetes are the two most abundant bacterial phyla under the experimental conditions, and the switch from a low-fat diet to a high-fat diet changed the composition of the mouse gut microbiota (Figure 4B-D). In addition, $300 \mathrm{mg} / \mathrm{kg} / \mathrm{day}$ gypenosides treatment significantly increased Verrucomicrobia content, which played a role in prebiotics (Figure 4A). The gut microbiota of the HFD group mice was characterized by a significant 3.2-fold increase of the Firmicutes to Bacteroidetes ratio compared with that of the low-fat diet group, and the ratio was decreased by $20 \%$ and $58.6 \%$ by the gypenoside treatment at a dose of 100 and $300 \mathrm{mg} / \mathrm{kg} /$ day, respectively $(P<0.05$, Figure 4D). Firmicutes are Gram-positive bacteria, which are involved in energy resorption and obesity. Bacteroidetes have been shown to exert immunomodulatory properties on the host. The change in the ratio of Firmicutes to Bacteroidetes may alter the weight gain or loss of the hosts. A high ratio of Frimcutes to Bacteroidetes has been found in obese mice and human adults compared to lean mice and human adults, respectively. ${ }^{17}$ Gypenosides treatment significantly decreased the ratio of Firmicutes to Bacteroidetes, which might be another possible mechanism involved in the effect of gypenoside in reducing the development of obesity and insulin resistance in HFD mice.

In addition, to identify the characteristic bacteria which were specific for each treatment group, LEfSe analysis based on the nonparametric factorial Kruskal-Wallis (KW) sum-rank test was applied. According to a pairwise comparison between the gut microbiota among the different treatment groups, high-dose gypenosides treatment significantly increased the abundance of genus Akkermansia and Lactobacillus and phylum Verrucomicrobia compared with the LFD and HFD control groups $(P<0.05$, Figure 5). Gypenosides significantly stimulated Akkermansia at a dose of $300 \mathrm{mg} / \mathrm{kg} /$ day. Akkermansia muciniphila (A. muciniphila), belonging to Verrucomicrobia, made a large contribution to the observed differences in the gut microbial compositions in the LFD, HFD, and HFD plus high dose of gypenosides mice $(P<0.05)$. A. muciniphila is a mucindegrading bacterium commonly found in human gut. $A$. muciniphila has been inversely associated with the risk of obesity, diabetes, inflammation, and metabolic disorders. ${ }^{15} A$. muciniphila may produce a number of fermentation products, including short-chain fatty acids (SCFA), which are substrates for gluconeogenesis and are involved in signaling to the host by activating G-protein-coupled receptors such as GPR41/43 and TGR-5. They could trigger the release of the hormone glucagon-like peptide-1(GLP-1), leading to an improved glucose homeostasis. Due to its beneficial activities against obesity and diabetes, $A$. muciniphila has drawn much attention for research and development in recent years. ${ }^{15,30,31}$ A number of human and animal studies have shown that the abundance of A. muciniphila in the gut can be enhanced through dietary interventions, $^{32}$ which has been considered a significant beneficial effect. The results from this study suggested that gypenosides might be a preferable prebiotics nutrient for $A$. muciniphila. Taken together, the data from this study suggested that altering gut microbiota might play an important role in an overall beneficial effect of gypenosides in reducing the development of obesity and the related insulin resistance.

This study showed that gypenoside could improve glycolipid metabolism and stimulate BAT activity, WAT browning, and lipid $\beta$-oxidation while decreasing the ratio of Firmicutes to Bacteroidetes and enhancing the abundance of Akkermansia muciniphila. These results suggested that gypenosides intake might serve as a possible approach to reduce the risk of the diet-induced overweight and insulin intolerance. In addition, gypenosides might prevent the HFD-induced obesity and insulin resistance through simultaneously promoting energy expenditure and modulating gut microbiota.

\section{ASSOCIATED CONTENT}

\section{S Supporting Information}

The Supporting Information is available free of charge on the ACS Publications website at DOI: 10.1021/acs.jafc.7b03382. 
Primer sequences for RT-PCR (PDF)

\section{AUTHOR INFORMATION}

\section{Corresponding Author}

*Tel: 301-0405-0761, Fax: 301-314-3313, E-mail: lyu5@umd. edu.

\section{ORCID $\odot$}

Qin Wang: 0000-0002-7496-3921

Liangli Lucy Yu: 0000-0001-6497-0864

\section{Notes}

The authors declare no competing financial interest.

\section{ACKNOWLEDGMENTS}

This research was supported by grants from the National High Technology Research and Development Program of China (Grants 2013AA102202 and 2013AA102207), a grant from the Beijing Advanced Innovation Center for Food Nutrition and Human Health, Beijing Technology \& Business University (BTBU), and the Beijing Excellent Talents Funding for the Youth Scientist Innovation Team.

\section{ABBREVIATIONS USED}

BAT, brown adipocyte tissue; WAT, white adipose tissue; UCP-1, uncoupling protein 1 ; PGC-1 $\alpha$, PPAR- $\gamma$ coactivator- $1 \alpha$; PRDM16, PR domain containing 16; Cide $\alpha$, cell deathinducing DNA fragmentation factor; Cox $7 \alpha$, cytochrome $c$ oxidase, subunit VII $\alpha$; Elovl3, long-chain fatty acids-like 3; CPT1, carnitine palmitoyl transterase-1; MCAD, medium-chain acyl-CoA dehydrogenase

\section{REFERENCES}

(1) Chevalier, C.; Stojanovic, O.; Colin, D. J.; Suarez-Zamorano, N.; Tarallo, V.; Veyrat-Durebex, C.; Rigo, D.; Fabbiano, S.; Stevanovic, A.; Hagemann, S.; Montet, X.; Seimbille, Y.; Zamboni, N.; Hapfelmeier, S.; Trajkovski, M. Gut microbiota orchestrates energy homeostasis during cold. Cell 2015, 163, 1360-1374.

(2) Cannon, B.; Nedergaard, J. Brown adipose tissue: function and physiological significance. Physiol. Rev. 2004, 84, 277-359.

(3) Cohen, P.; Spiegelman, B. M. Brown and beige fat: molecular parts of a thermogenic machine. Diabetes 2015, 64, 2346-2351.

(4) Zhang, X.; Zhang, Q. X.; Wang, X.; Zhang, L.; Qu, W.; Bao, B.; Liu, C. A.; Liu, J. Dietary luteolin activates browning and thermogenesis in mice through an AMPK/PGC1 alpha pathwaymediated mechanism. Int. J. Obes. 2016, 40, 1841-1849.

(5) Kim, K. H.; Park, Y. Food components with anti-obesity effect. Annu. Rev. Food Sci. Technol. 2011, 2, 237-257.

(6) Yang, F.; Shi, H. M.; Zhang, X. W.; Yu, L. L. Two novel antiinflammatory 21-nordammarane saponins from tetraploid jiaogulan (Gynostemma pentaphyllum). J. Agric. Food Chem. 2013, 61, 1264612652.

(7) Park, S. H.; Huh, T. L.; Kim, S. Y.; Oh, M. R.; Tirupathi Pichiah, P. B.; Chae, S. W.; Cha, Y. S. Antiobesity effect of Gynostemma pentaphyllum extract (actiponin): a randomized, double-blind, placebocontrolled trial. Obesity 2014, 22, 63-71.

(8) Megalli, S.; Davies, N. M.; Roufogalis, B. D. Anti-hyperlipidemic and hypoglycemic effects of Gynostemma pentaphyllum in the Zucker fatty rat. I. Pharm. Pharm. Sci. 2006, 9, 281-291.

(9) Yeo, J.; Kang, Y. J.; Jeon, S. M.; Jung, U. J.; Lee, M. K.; Song, H.; Choi, M. S. Potential hypoglycemic effect of an ethanol extract of gynostemma pentaphyllum in C57BL/KsJ-db/db Mice. J. Med. Food 2008, 11, 709-716.

(10) Yang, F.; Shi, H. M.; Zhang, X. W.; Yang, H. S.; Zhou, Q.; Yu, L. L. Two new saponins from tetraploid jiaogulan (Gynostemma pentaphyllum), and their anti-inflammatory and alpha-glucosidase inhibitory activities. Food Chem. 2013, 141, 3606-3613.
(11) Hu, Y. M.; Ip, F. C. F.; Fu, G. M.; Pang, H. H.; Ye, W. C.; Ip, N. Y. Dammarane saponins from Gynostemma pentaphyllum. Phytochemistry 2010, 71, 1149-1157.

(12) Hsu, H. Y.; Yang, J. S.; Lu, K. W.; Yu, C. S.; Chou, S. T.; Lin, J. J.; Chen, Y. Y.; Lin, M. L.; Chueh, F. S.; Chen, S. S.; Chung, J. G. An experimental study on the antileukemia effects of gypenosides in vitro and in vivo. Integr. Cancer Ther. 2011, 10, 101-112.

(13) Gauhar, R.; Hwang, S. L.; Jeong, S. S.; Kim, J. E.; Song, H.; Park, D. C.; Song, K. S.; Kim, T. Y.; Oh, W. K.; Huh, T. L. Heat-processed Gynostemma pentaphyllum extract improves obesity in $o b / o b$ mice by activating AMP-activated protein kinase. Biotechnol. Lett. 2012, 34, $1607-1616$

(14) Liu, J.; Yang, P. Y.; Shi, H. M.; Sun, X. J.; Lee, S. H.; Yu, L. L. A novel Gynostemma pentaphyllum saponin and its adipogenesis inhibitory effect through modulating Wnt/beta-catenin pathway and cell cycle in mitotic clonal expansion. J. Funct. Foods 2015, 17, 552562 .

(15) Dao, M. C.; Everard, A.; Aron-Wisnewsky, J.; Sokolovska, N.; Prifti, E.; Verger, E. O.; Kayser, B. D.; Levenez, F.; Chilloux, J.; Hoyles, L.; Consortium, M. I.-O.; Dumas, M. E.; Rizkalla, S. W.; Dore, J.; Cani, P. D.; Clement, K. Akkermansia muciniphila and improved metabolic health during a dietary intervention in obesity: relationship with gut microbiome richness and ecology. Gut 2016, 65, 426-436.

(16) Chang, C. J.; Lin, C. S.; Lu, C. C.; Martel, J.; Ko, Y. F.; Ojcius, D. M.; Tseng, S. F.; Wu, T. R.; Chen, Y. Y.; Young, J. D.; Lai, H. C. Ganoderma lucidum reduces obesity in mice by modulating the composition of the gut microbiota. Nat. Commun. 2015, 6, 7489.

(17) Turnbaugh, P. J.; Ley, R. E.; Mahowald, M. A.; Magrini, V.; Mardis, E. R.; Gordon, J. I. An obesity-associated gut microbiome with increased capacity for energy harvest. Nature 2006, 444, 1027-1031.

(18) Sommer, F.; Backhed, F. The gut microbiota-masters of host development and physiology. Nat. Rev. Microbiol. 2013, 11, 227-238.

(19) Liu, J.; Li, Y. F.; Shi, H. M.; Wang, T.; Wu, X. L.; Sun, X. J.; Yu, L. L. Components characterization of total tetraploid jiaogulan (Gynostemma pentaphyllum) saponin and its cholesterol-lowering properties. J. Funct. Foods 2016, 23, 542-555.

(20) Chang, W. C.; Wu, J. S.; Chen, C. W.; Kuo, P. L.; Chien, H. M.; Wang, Y. T.; Shen, S. C. Protective effect of vanillic acid against hyperinsulinemia, hyperglycemia and hyperlipidemia via alleviating hepatic insulin resistance and inflammation in high-fat diet (HFD)-fed rats. Nutrients 2015, 7, 9946-9959.

(21) Huang, J. C.; Lin, X. H.; Xue, B.; Luo, J. M.; Gao, L. J.; Wang, Y.; Ou, S. Y.; Peng, X. C. Impact of polyphenols combined with highfat diet on rats' gut microbiota. J. Funct. Foods 2016, 26, 763-771.

(22) Seale, P.; Conroe, H. M.; Estall, J.; Kajimura, S.; Frontini, A.; Ishibashi, J.; Cohen, P.; Cinti, S.; Spiegelman, B. M. Prdm16 determines the thermogenic program of subcutaneous white adipose tissue in mice. J. Clin. Invest. 2011, 121, 96-105.

(23) Ma, X.; Xu, L.; Alberobello, A. T.; Gavrilova, O.; Bagattin, A.; Skarulis, M.; Liu, J.; Finkel, T.; Mueller, E. Celastrol protects against obesity and metabolic dysfunction through activation of a HSF1PGC1alpha transcriptional axis. Cell Metab. 2015, 22, 695-708.

(24) Poher, A. L.; Altirriba, J.; Veyrat-Durebex, C.; RohnerJeanrenaud, F. Brown adipose tissue activity as a target for the treatment of obesity/insulin resistance. Front. Physiol. 2015, 6, 1-9.

(25) Fisher, F. M.; Kleiner, S.; Douris, N.; Fox, E. C.; Mepani, R. J.; Verdeguer, F.; Wu, J.; Kharitonenkov, A.; Flier, J. S.; Maratos-Flier, E.; Spiegelman, B. M. FGF21 regulates PGC-1alpha and browning of white adipose tissues in adaptive thermogenesis. Genes Dev. 2012, 26, 271-281.

(26) Cohen, P.; Levy, J. D.; Zhang, Y.; Frontini, A.; Kolodin, D. P.; Svensson, K. J.; Lo, J. C.; Zeng, X.; Ye, L.; Khandekar, M. J.; Wu, J.; Gunawardana, S. C.; Banks, A. S.; Camporez, J. P.; Jurczak, M. J.; Kajimura, S.; Piston, D. W.; Mathis, D.; Cinti, S.; Shulman, G. I.; Seale, P.; Spiegelman, B. M. Ablation of PRDM16 and beige adipose causes metabolic dysfunction and a subcutaneous to visceral fat switch. Cell 2014, 156, 304-316.

(27) Broeders, E. P. M.; Nascimento, E. B. M.; Havekes, B.; Brans, B.; Roumans, K. H. M.; Tailleux, A.; Schaart, G.; Kouach, M.; Charton, 
J.; Deprez, B.; Bouvy, N. D.; Mottaghy, F.; Staels, B.; Lichtenbelt, W. D. V.; Schrauwen, P. The bile acid chenodeoxycholic acid increases human brown adipose tissue activity. Cell Metab. 2015, 22, 418-426.

(28) Thomas, C.; Gioiello, A.; Noriega, L.; Strehle, A.; Oury, J.; Rizzo, G.; Macchiarulo, A.; Yamamoto, H.; Mataki, C.; Pruzanski, M.; Pellicciari, R.; Auwerx, J.; Schoonjans, K. TGR5-mediated bile acid sensing controls glucose homeostasis. Cell Metab. 2009, 10, 167-177.

(29) Arora, T.; Backhed, F. The gut microbiota and metabolic disease: current understanding and future perspectives. J. Intern. Med. 2016, 280, 339-349.

(30) Schneeberger, M.; Everard, A.; Gomez-Valades, A. G.; Matamoros, S.; Ramirez, S.; Delzenne, N. M.; Gomis, R.; Claret, M.; Cani, P. D. Akkermansia muciniphila inversely correlates with the onset of inflammation, altered adipose tissue metabolism and metabolic disorders during obesity in mice. Sci. Rep. 2015, 5, 1-14.

(31) Everard, A.; Belzer, C.; Geurts, L.; Ouwerkerk, J. P.; Druart, C.; Bindels, L. B.; Guiot, Y.; Derrien, M.; Muccioli, G. G.; Delzenne, N. M.; De Vos, W. M.; Cani, P. D. Cross-talk between Akkermansia muciniphila and intestinal epithelium controls diet-induced obesity. Proc. Natl. Acad. Sci. U. S. A. 2013, 110, 9066-9071.

(32) Zhou, K. Q. Strategies to promote abundance of Akkermansia muciniphila, an emerging probiotics in the gut, evidence from dietary intervention studies. J. Funct. Foods 2017, 33, 194-201. 